Further investigations including genetic analysis revealed mutaion in NFKB2 protein (heterozygous c $2557>\mathrm{T}$ ). Parental blood samples too were taken for genetic studies. They are non consaguineous.

The diagnosis was mutation in NFKB2 leading to Common Variable Immunodeficiency (CVID) phenotype associated with hypogammaglobulinaemia, alopecia and onycodystrophy. Because of close relationship with endocrinopathies he was referred to endocrine team.

$\mathrm{He}$ is on prophylactic azithromycin. Protopic ointment $0.1 \%$ aplied topically to scalp and nails. Immunology team suggested that he will need immunoglobulin replacement therapy in the future. He may require oral immunosuppressants if the response to the recommended therapy is inadequate.

Discussion Our patient with alopecia, nail dystrophy and hypogammaglobulinaemia has genetic abnormality with mutation NFKB2 protein. There were previous reported patients carrying NFKB2 mutation demonstrated endocrinopathies in addition to ectodermal abnormalities together with other autoimmune disordres such as vitiligo and auto immune thrombocytopoenic purpura. Therefore the patient we reported also has been directed to rule out these endocrinopathies especially adrenocortical deficiencies and auto immune disorders.

Conclusion Patients with alopecia, ectodermal dysplasia should be investigated and monitored thoroughly in order to confirm or exclude the involvements with other disorders such as hypogammaglobulinaemia, genetic abnormalities (CVID) and it's links with endocrinopathies and auto immune disorders.

\section{P109 AN UNUSUAL CASE OF AN ANTENATAL DIAGNOSIS OF HUGE NECK MASS; A THYROID IMMATURE TERATOMA IN A NEWBORN}

${ }^{1}$ Ranya Abuhaleeqa*, 'Susan O'Connell, ${ }^{1}$ Rania Mehanna, ${ }^{1} J o h n$ Russell, ${ }^{2}$ Colm O'Donnell. 'Our Lady's Children Hospital Crumlin, Dublin, Ireland; ${ }^{2}$ National Maternity Hospital, Dublin, Ireland

\subsection{6/archdischild-2019-epa.464}

Introduction Head neck teratomas are rare benign tumors consisting of $3-5 \%$ of all teratomas $(1,2)$. Teratomas are embryonal neoplasms that arise when totipotential germ cells escape the developmental control of primary organizers and give rise to more or less organoid masses in which tissues derived from all three blastodermic layers (ectoderm, endoderm, and mesoderm) can be identified. Their histologic features are therefore heterogenous and may include cystic or solid areas with organoid patterns as well as mature or immature components.

Case description We report a case of an antenatal diagnosis of a huge neck mass on antenatal ultrasound and fetal in utero MRI at 33 weeks gestation. He was born by ELSCS at 38 weeks gestation and an endocotracheal intubation was performed directly to maintain airway. The MRI neck postnatal confirmed a well circumscribed multicystic and solid anterior neck mass $9.7 \times 5.2 \times 4.9 \mathrm{~cm}$. Surgical resection of the mass and thymectomy were done on day 2 after birth. The thyroid gland was not identified surgically. A single parathyroid gland was identified intraoperatively and preserved. The pathology report of the neck mass confirmed immature teratoma arising within and largely effacing the thyroid gland. No malignant germ cell tumour elements identified. $\mathrm{He}$ required levothyroxine treatment to replace the thyroid function. Hypocalcaemia developed postsurgical likely as a result of hypoparathyroidism. He was therefore managed by one alpha and calcium supplements to maintain normocalcaemia. He is feeding orally and continues to show appropriate normal growth and development.

Discussion Cervical teratomas in children are almost always benign but locally are aggressive. They can present with respiratory airway compromise and excision is required. A multidisciplinary management is needed with multiple specialties involvement. If complete thyroid tissue is removed replacement therapy is required post operatively. Risk of hypocalcemia secondary to hypoparathyroidism developing post operatively should be monitored and treated accordingly.

\section{REFERENCES}

1. Rothschild MA, Catalano P, Urken M, Brandwein M, Som P, Norton K, Biller HF. Evaluation and management of congenital cervical teratoma. Case report and review. Arch Otolaryngol Head Neck Surg. 1994;120(4):444-448.

2. Lack EE. Extragonadal germ cell tumors of the head and neck region: review of 16 cases. Hum Pathol. 1985;16:56-64.

\section{P110 THE BOY WITH MOYAMOYA: STROKES AND A WISP OF SMOKE}

C Dyson*, K Flinn, E O’ Mahony, AM Murphy. Department of Paediatrics, University Hospital Limerick, Limerick, Ireland

\subsection{6/archdischild-2019-epa.465}

Aims Moyamoya is a rare progressive occlusive cerebrovascular disease, affecting both children and adults in a bimodal age pattern. The peak incidence in children is 7-10 years old and can present with a variety of clinical scenarios.

Our aim is to describe the clinical presentation, management and outcome to date of a now 5 year old boy who was diagnosed with Moya Moya at 22 months of age.

Methods We report the presenting features, examinations findings and results of radiological images and laboratory investigations, treatment and natural history with regard to our now school age patient.

Results A 22 month old boy presented to the ED following a fall to one side and an episode of high pitched crying. $\mathrm{He}$ was afebrile with no recent temperatures or illnesses. His history was notable for a previous afebrile seizure at five months of age lasting over one hour, and recent concerns regarding developmental delay. He had previously had a normal MRI and CT brain, and a normal EEG.

The seizure was eventually controlled on phenobarbitone, and an urgent CT brain showed frontal lobe ischaemia with chronic infarct in the left parietal lobe. He was transferred to Temple St Hospital and subsequently diagnosed with moyamoya. He attended Great Ormond Street Children's Hospital for several revascularisation surgeries.

He was diagnosed with autism and a sensory processing disorder, and is fed via a gastrostomy tube.

$\mathrm{He}$ is now five years old, and has been seizure free for several years. He is ambulant, with over 100 words and good comprehension.

Conclusions Our case highlights the success of multidisplinary input, sophisticated sub-specialist care, international expertise and devoted parenting in contributing to the quality of life, despite this devastating disease, in our young patient to date. 